\title{
Dynamic installation, keying and diving of OMNI-Max anchors in clay
}

\author{
Y. H. KIM* and M. S. HOSSAIN*
}

\begin{abstract}
OMNI-Max anchors are designed with the aim of diving upon pullout, gaining capacity with increasing pullout distance. However, this diving potential is yet to be confirmed; that is, the critical question to be resolved is whether or not the anchor dives upon pullout and, if so, in what conditions. This paper reports the results from three-dimensional dynamic finite-element analysis undertaken to provide insight into the behaviour of OMNI-Max anchors during pullout in non-homogeneous clay. Largedeformation finite-element (LDFE) analyses were carried out using the coupled Eulerian-Lagrangian approach, modifying the simple elastic-perfectly plastic Tresca soil model to allow strain softening, and to incorporate strain-rate dependency of the shear strength. The keying process and diving patterns during anchor pullout revealed three governing factors including: (a) pullout inclination angle; (b) padeye offset ratio; (c) final installation depth. For designing anchors to dive into deeper, it was recommended that the anchor loading angle to the horizontal at the padeye should be $45^{\circ}$ or less and the anchor padeye offset ratio should be in the range of $0.25 \sim 0.53$ (offset angle of $14 \sim 28^{\circ}$ ).
\end{abstract}

KEYWORDS: anchors \& anchorages; clays; numerical modelling; offshore engineering

\section{INTRODUCTION}

The OMNI-Max anchor is the most recent generation of dynamically installed anchors (DIAs) for mooring floating facilities for deep-water oil and gas developments. In contrast to typical torpedo-shaped DIAs, the OMNI-Max anchor features an arm that transfers the loading point nearer to the head of the anchor (see Fig. 1). It was anticipated that this configuration would force the anchor to dive deeper when pulled, gradually increasing the capacity (Shelton, 2007; Zimmerman et al., 2009; Nie \& Shelton, 2011; Shelton et al., 2011). If the anchor dives deeper, the geotechnical resistance increases and the capacity is eventually governed by the tension capacity of the mooring line.

Investigations on OMNI-Max anchors are sparse. Recently, the problem has been addressed through numerical analysis, although limited to pre-embedded anchors. Liu et al. (2014) and Wei et al. (2015) carried out largedeformation finite-element (LDFE) analyses and plasticity analyses. The clay was modelled as rate-independent and non-softening ideal Tresca material.

Kim \& Hossain (2015) recently developed a numerical framework for undertaking three-dimensional LDFE analysis on dynamic installation of OMNI-Max anchors accounting for frictional resistance along the surfaces of the anchor, strain rate dependency and gradient of the soil undrained shear strength. This framework was incorporated in the present study for the monotonic pullout of anchors with the aim of investigating the keying process and diving potential.

\section{NUMERICAL ANALYSIS \\ Analysis details \\ Three-dimensional LDFE analyses were carried out using the coupled Eulerian-Lagrangian (CEL) approach in}

Manuscript received 15 February 2016; revised manuscript accepted 8 August 2016. Published online ahead of print 19 September 2016. Discussion on this paper closes on 1 June 2017, for further details see $\mathrm{p}$. ii.

* Centre for Offshore Foundation Systems (COFS), The University of Western Australia, Crawley, WA, Australia. the commercial finite-element package Abaqus/Explicit (Version 6.12 (Dassault Systèmes, 2012)). Extensive background information about installation modelling of the OMNI-Max anchor can be found in Kim \& Hossain (2015), which is not repeated here.

Considering the symmetry of the problem, only one half of an anchor and soil domain were modelled. The lateral extension of the soil domain was $55 D_{\mathrm{p}}$ from the centre of the anchor $\left(D_{\mathrm{p}}\right.$ is the anchor frontal projected area $\left(A_{\mathrm{p}}\right)$ equivalent diameter) on the pullout loading direction and $17 D_{\mathrm{p}}$ on the opposite direction. The height of the soil domain was $\sim 6 \cdot 6 L_{\mathrm{A}}$ to avoid the boundary effect during dynamic installation and diving process (as obtained from preliminary convergence studies; e.g. Kim et al. (2014); Kim \& Hossain (2015)).

A typical mesh is shown in Fig. 2. A very fine soil mesh was necessary to capture the anchor-soil contact accurately. Therefore, mesh convergence studies were first performed to ensure that the mesh was sufficiently fine to give accurate results. As shown in Fig. 3(a), five different mesh densities were considered for an OMNI-Max anchor installation ('very fine mesh zone' in Fig. 2) under an identical impact velocity of $v_{\mathrm{i}}=19 \mathrm{~m} / \mathrm{s}$. The numerical results based on mesh 1 and mesh 2, with minimum element sizes $\left(h_{\min }\right) 0 \cdot 15 t_{\mathrm{F}}$ and $0 \cdot 18 t_{\mathrm{F}}$, respectively (where $t_{\mathrm{F}}$ is the fin thickness), are essentially identical, indicating that mesh convergence was achieved with the density of mesh $2\left(h_{\min }=0 \cdot 18 t_{\mathrm{F}}\right)$. For the continuous inclined pullout simulation, again five different mesh densities of the dragging area ('fine mesh zone' in Fig. 2) were tested at the same pullout angle $\left(\theta_{\mathrm{a}}=30^{\circ}\right)$ and padeye offset ratio $(\eta=0 \cdot 35)$. As shown in Fig. 3(b), mesh convergence was achieved with a slightly larger element size of $0 \cdot 5 t_{\mathrm{F}}$ (mesh 2) compared to vertical installation. As such, for subsequent analyses, the typical minimum soil element size along the trajectory of the anchor was selected as $0 \cdot 18 t_{\mathrm{F}}$ for vertical installation (very fine mesh zone) and $0 \cdot 5 t_{\mathrm{F}}$ for inclined pullout (fine mesh zone). Total number of elements in the whole soil domain was around 7240000 . The anchor was simplified as a rigid body.

The simulation was fully integrated taking into account the disturbed soil conditions during the installation of the 


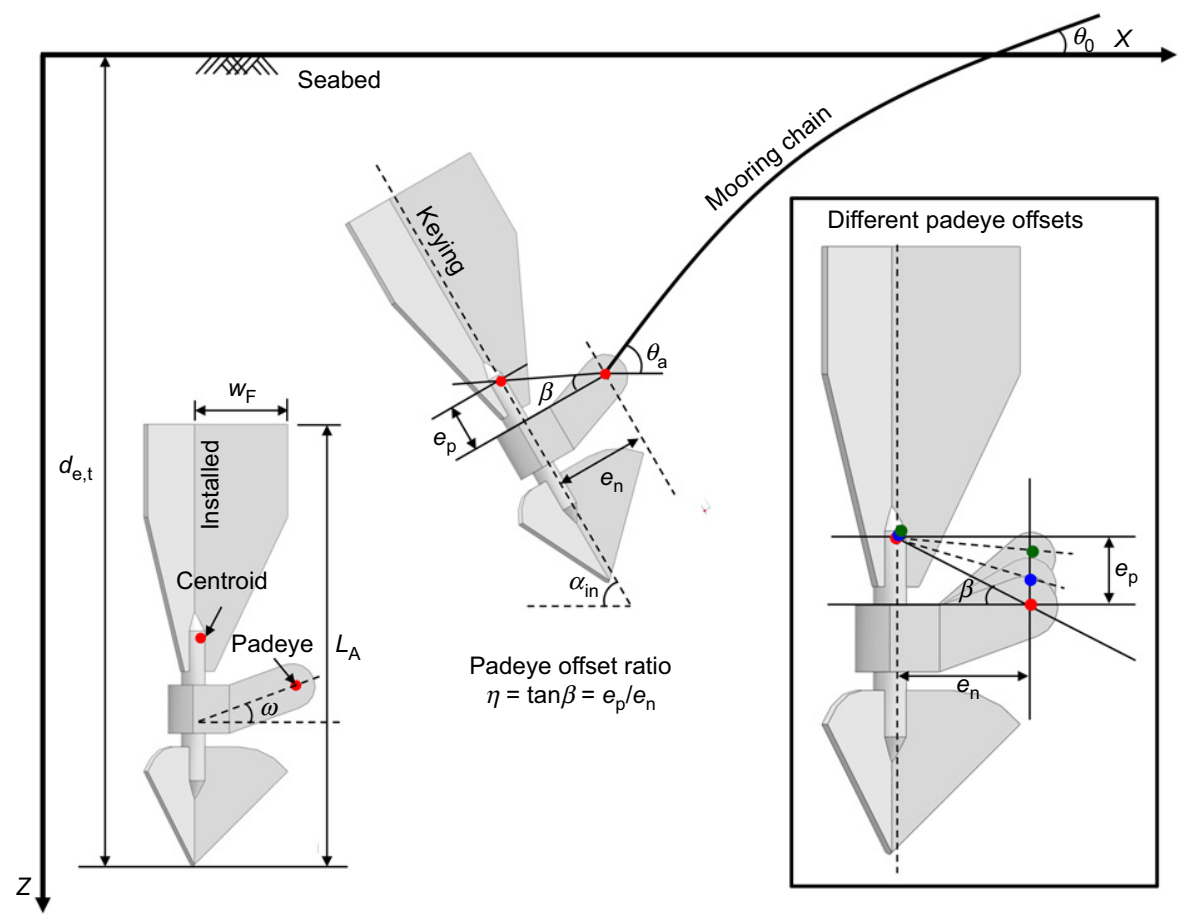

Fig. 1. Schematic diagram of installed anchor and subsequent rotation upon pullout

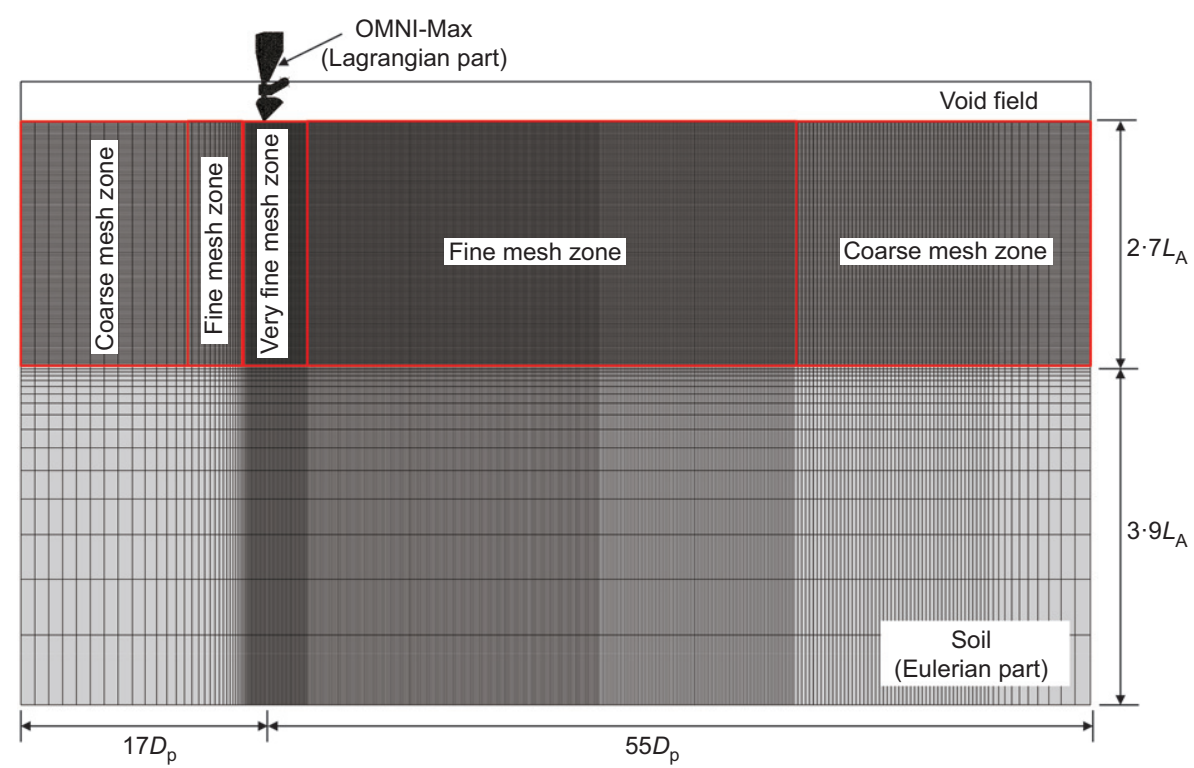

Fig. 2. Typical mesh used in LDFE analysis

anchor for the pullout stage. In this study, an inclined pullout loading, rather than an inclined displacement, was applied to the anchor padeye $\left(\theta_{\mathrm{a}}\right)$ to obtain apparent anchor trajectory. The dynamic installation and monotonic pullout of the anchors in clay are completed under undrained conditions. The soil was thus modelled as an elasto-plastic material obeying a Tresca yield criterion, but extended as described later to capture strain-rate and strain-softening effects. A user subroutine was implemented to track the evolving soil strength profile. The elastic behaviour was defined by a Poisson ratio of 0.49 and Young's modulus of $500 s_{\mathrm{u}}$ throughout the soil profile. Total stress analyses were carried out adopting a uniform effective unit weight of $6 \mathrm{kN} / \mathrm{m}^{3}$ over the soil depth, representing a typical average value for field conditions.
The Tresca soil model was extended to capture strain-rate and strain-softening effects, following the models of Einav-Randolph (Einav \& Randolph, 2005).

$$
\begin{aligned}
s_{\mathrm{u}}= & \left\{1+\mu \log \left[\frac{\max \left(|\dot{\gamma}|, \dot{\gamma}_{\text {ref }}\right)}{\dot{\gamma}_{\text {ref }}}\right]\right\} \\
& \times\left[\delta_{\text {rem }}+\left(1-\delta_{\text {rem }}\right) \mathrm{e}^{-3 \xi / \xi_{95}}\right] s_{\mathrm{u}, \text { ref }}
\end{aligned}
$$

where $s_{\mathrm{u}, \text { ref }}$ is the shear strength at the reference shear strain rate of $\dot{\gamma}_{\text {ref }}$. The first bracketed term of equation (1) augments the strength according to the maximum strain rate, $\dot{\gamma}$, relative to a reference value, $\dot{\gamma}_{\text {ref }}$, which was considered as $1.5 \% / \mathrm{h}$ corresponding to the typical strain rate in triaxial tests (Lunne \& Andersen, 2007). The augmentation of shear 


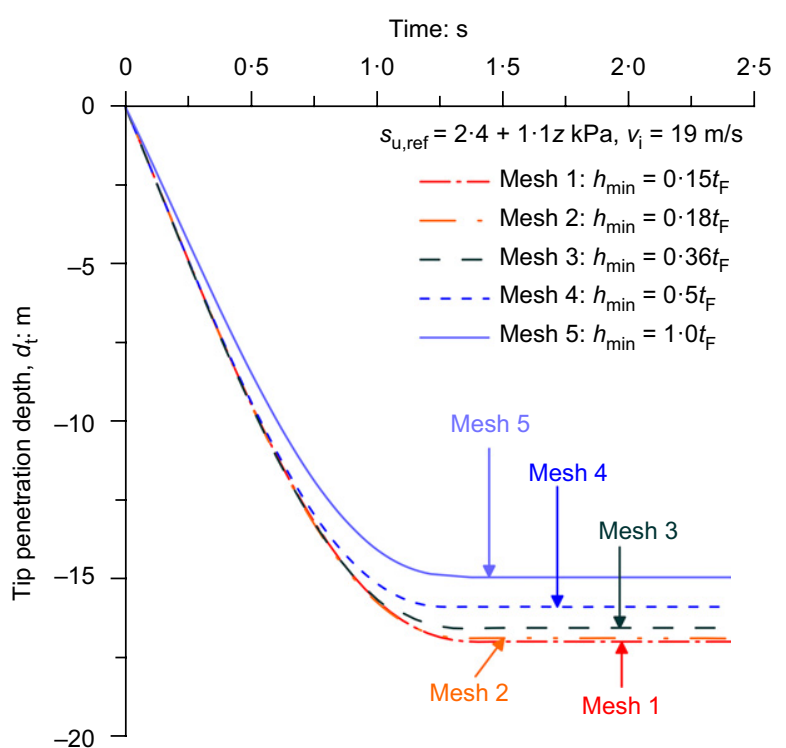

(a)

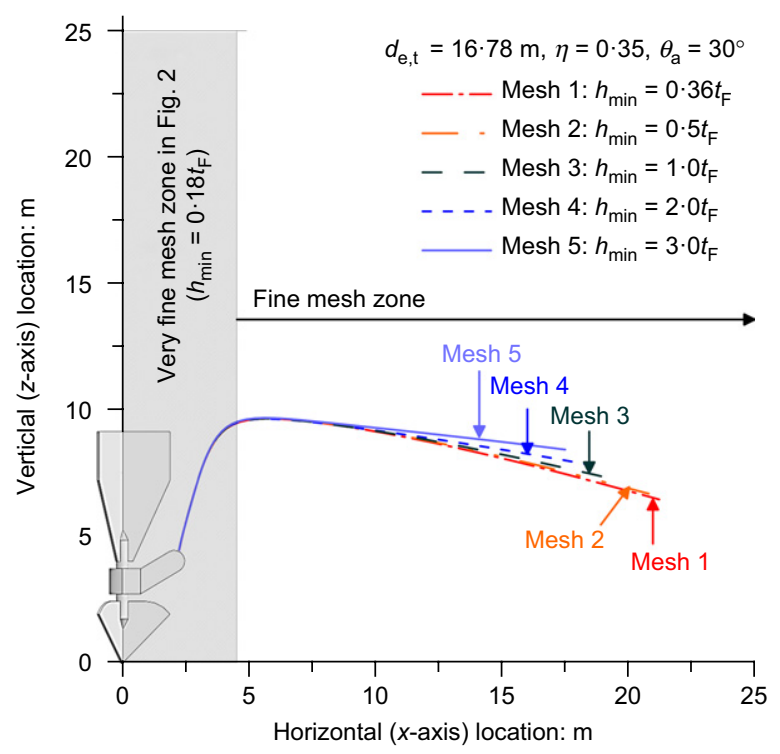

(b)

Fig. 3. Mesh convergence studies of OMNI-Max anchor: (a) anchor installation; (b) anchor pullout (followed by installation)

strength follows a logarithmic law with rate parameter $\mu$ assumed as $0 \cdot 1$ (Low et al., 2008). The second part of equation (1) models the degradation of strength according to an exponential function of cumulative plastic shear strain, $\xi$, from the intact condition to a fully remoulded ratio, $\delta_{\text {rem }}$ (the inverse of the sensitivity, $S_{\mathrm{t}}$ ). The relative ductility is controlled by the parameter, $\xi_{95}$, which represents the cumulative plastic shear strain required for $95 \%$ remoulding. A typical value of $\xi_{95}=20$ (i.e. $2000 \%$ shear strain; Randolph, 2004) was considered, as was also adopted in previous studies on DIAs (Kim \& Hossain, 2015; Kim et al., 2015a, 2015b). Further details can be found in Hossain \& Randolph (2009) and Zheng et al. (2015).

The soil-anchor interface was modelled as frictional contact, using a general contact algorithm and specifying a (total stress) Coulomb friction law together with a limiting shear stress $\left(\tau_{\max }\right)$ along the anchor-soil interface (Ma et al., 2014). For each case, the limiting interface friction was determined by setting $\tau_{\max }$ equal to an interface friction ratio, $\alpha$, times the average $s_{\mathrm{u}}$ along the anchor length, with $\alpha$ taken as the inverse soil sensitivity, $1 / S_{\mathrm{t}}$. Typical computation times on a high-performance workstation with 12 central processing unit cores were about 10 days for an anchor dynamic installation followed by monotonic pullout of $\sim 2.5$ anchor lengths.

\section{Different padeye offset}

Previous studies for the OMNI-Max anchor (Liu et al., 2014; Wei et al., 2015) did not simulate the anchor's loading arm. This study modelled the arm as a solid object. This is worth considering because of its proportion of volume and weight $(20 \%$ of the anchor). Fig. 1 defines the problem. The left-hand diagram shows that $d_{\mathrm{e}, \mathrm{t}}$ is the anchor tip embedment depth after installation, with the geometric centroid and padeye labelled. For the dynamic installation, the projected area $\left(A_{\mathrm{p}}\right)$ and submerged anchor weight $\left(W_{\mathrm{s}}\right)$ of the anchor were fixed. With those fixed values, the final installed (embedment) depth $\left(d_{\mathrm{e}, \mathrm{t}}\right)$ will be similar for a given impact velocity (Kim et al., 2015a).

Upon pullout (see the middle figure), the mooring chain forms an angle to the horizontal $\theta_{0}$ at the mudline and $\theta_{\mathrm{a}}$ at the padeye. $e_{\mathrm{n}}$ denotes the padeye eccentricity and $e_{\mathrm{p}}$ represents the padeye offset relative to the anchor centroid point. Tian et al. $(2014,2015)$ proposed a definition of the padeye offset ratio $\eta$ for the plate anchor as $e_{\mathrm{p}} / e_{\mathrm{n}}$ and highlighted its influence on the tendency to dive under loading. Assuming a constant anchor chain pulling angle, they showed that an optimal choice of $\eta$ can cause the anchor to dive, increasing embedment. Here, that concept was adopted and validated for the OMNI-Max anchor. The range of anchor dimensions considered is summarised in Table 1.

\section{VALIDATION AGAINST CENTRIFUGE TEST DATA}

Details of failure mechanisms during installation of different DIA configurations have been documented by Kim et al. (2015a, 2015b) and Kim \& Hossain (2015). Results in the following sections will focus mainly on the pullout behaviour of the OMNI-Max anchor.

\section{Comparison between $L D F E$ result and centrifuge test data}

The LDFE results were validated against centrifuge test data. Gaudin et al. (2013) presented data from a centrifuge test carried out at $200 \mathrm{~g}$ in kaolin clay $\left(S_{\mathrm{t}}=2 \cdot 4\right)$. The soil undrained shear strength of $s_{\mathrm{u}, \mathrm{ref}}=3+1.1 z \mathrm{kPa}$ was deduced from T-bar penetration tests. The model anchor (anchor A1; Table 1) was installed statically through jacking up to an embedment depth of $d_{\mathrm{e}, t} / L_{\mathrm{A}}=1.4$ and then pulled out monotonically at an angle of $0^{\circ}$ at the mudline $\left(\theta_{0}=0^{\circ}\right)$. The padeye offset ratio $\eta$ was $0 \cdot 65$. In the LDFE simulation, these parameters and $\mu=0 \cdot 1, \delta_{\text {rem }}=1 / S_{\mathrm{t}}=1 / 2 \cdot 4, \xi_{95}=20$ and $\dot{\gamma}_{\text {ref }}=1.5 \% / \mathrm{h}$ (as discussed in the earlier section entitled 'Analysis details') were considered. The pullout angle at the padeye $\left(\theta_{\mathrm{a}}\right)$ was adopted as $15^{\circ}$ following the Neubecker \& Randolph (1995) approach.

Figure 4(a) shows the computed and measured loaddisplacement curves. Both loading curves exhibit similar stages, including (a) load application (from point 1 to point 2): the mooring line is tightened and the pullout load develops rapidly; (b) anchor keying (from point 2 to point 3 ): the anchor starts keying (including rotating and translating) and the pullout load gradually increases; (c) anchor diving (after point 3): the steady load increase corresponds to the anchor diving into the soil. As a result, the anchor capacity increases steadily after the keying process. The anchor pullout load from the LDFE analysis is slightly (around $10 \%$ ) lower than the centrifuge test. This is because the load 
Table 1. Omni-Max anchor details

\begin{tabular}{|c|c|c|c|c|c|c|c|c|}
\hline Description & Symbol (unit) & $\mathrm{A} 1 *$ & $\mathrm{~A} 2 \dagger$ & A3 & A4 & A5 & A6 & A7\$ \\
\hline Total anchor length & $L_{\mathrm{A}}: \mathrm{m}$ & $9 \cdot 05$ & $9 \cdot 15$ & & & & & \\
\hline Fin thickness & $t_{\mathrm{F}}: \mathrm{m}$ & $0 \cdot 284$ & $0 \cdot 1$ & & & & & \\
\hline Fin width & $w_{\mathrm{F}}: \mathrm{m}$ & $1 \cdot 886$ & 1.96 & & & & & \\
\hline $\begin{array}{l}\text { Anchor frontal projected area } \\
\text { equivalent diameter }\end{array}$ & $D_{\mathrm{p}}: \mathrm{m}$ & $1 \cdot 71$ & $1 \cdot 56$ & & & & & \\
\hline Anchor volume & $V_{\mathrm{A}}: \mathrm{m}^{3}$ & $9 \cdot 62$ & $5 \cdot 02$ & & & & & \\
\hline Anchor dry weight & $W_{\mathrm{d}}: \mathrm{kN}$ & $802 \cdot 1$ & 390 & & & & & \\
\hline Anchor submerged weight & $W_{\mathrm{s}}: \mathrm{kN}$ & 706 & 341 & & & & & \\
\hline Padeye offset & $e_{\mathrm{p}}: \mathrm{m}$ & $1 \cdot 08$ & $0 \cdot 19$ & $0 \cdot 277$ & $0 \cdot 503$ & $0 \cdot 684$ & $0 \cdot 994$ & $1 \cdot 544$ \\
\hline Padeye eccentricity & $e_{n}: \mathrm{m}$ & $1 \cdot 651$ & $2 \cdot 00$ & $2 \cdot 035$ & 1.99 & 1.96 & 1.89 & $2 \cdot 057$ \\
\hline Offset angle & $\beta:$ degrees & $33 \cdot 2$ & $5 \cdot 4$ & $7 \cdot 75$ & $14 \cdot 2$ & $19 \cdot 3$ & $27 \cdot 8$ & $36 \cdot 89$ \\
\hline Padeye offset ratio & & 0.65 & $0 \cdot 1$ & $0 \cdot 136$ & $0 \cdot 253$ & $0 \cdot 35$ & $0 \cdot 526$ & 0.75 \\
\hline Padeye inclination & $\omega:$ degrees & 40 & 50 & 40 & 35 & 30 & & 20 \\
\hline
\end{tabular}

*Centrifuge testing model anchor (Gaudin et al., 2013).

$\dagger$ Field testing prototype anchor (Zimmerman et al., 2009).

$\$$ Weightless padeye (same geometry with A6).

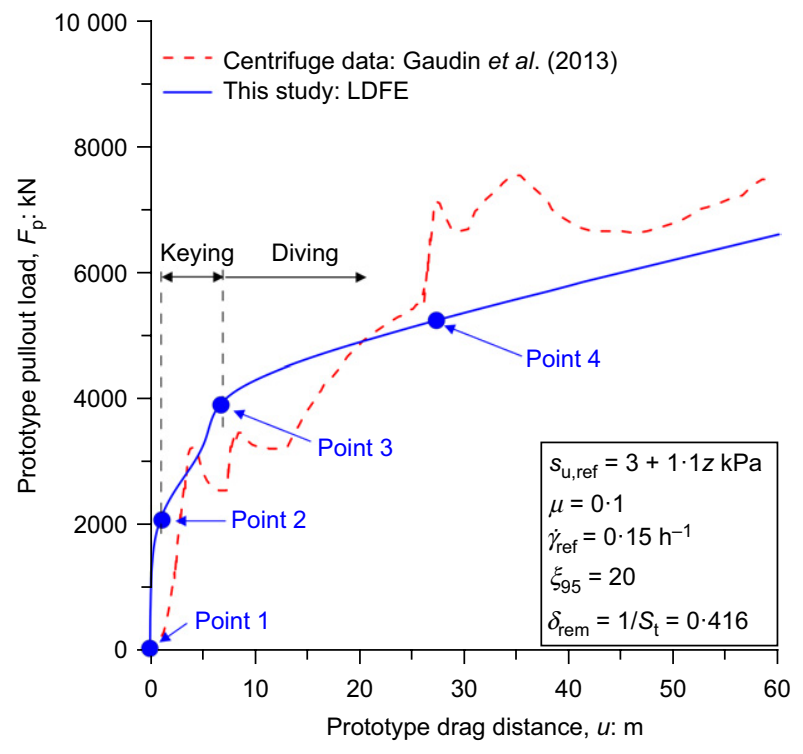

(a)

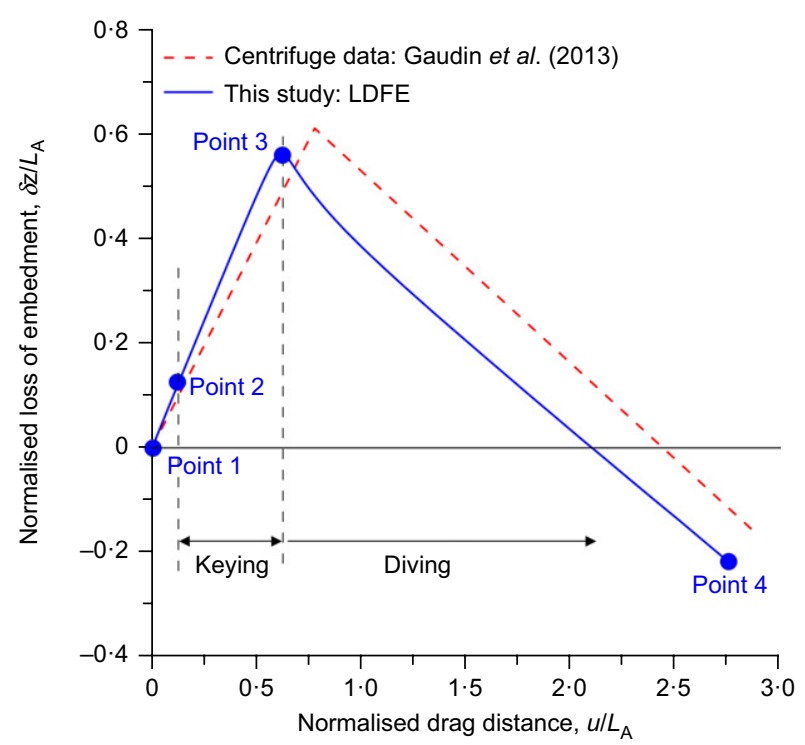

(b)

Fig. 4. Comparison between LDFE results and measured data: (a) pullout load-displacement curve; (b) anchor trajectories at padeye increases occurred at $u=7 \mathrm{~m}$ and $26 \mathrm{~m}$ (where $u$ is the drag distance) due to pauses of loading and ramping downramping up of the centrifuge. Each pause was followed by a significant load increase, due to the increase in soil strength as a result of reconsolidation of the soil (Gaudin et al., 2013). Fig. 4(b) also shows the anchor trajectory during pullout. Note that the anchor embedment is represented by the padeye position. Good agreement can be seen with the measured anchor trajectory and diving effect; the slight discrepancy in terms of the location of point 3 may be associated with the additional consolidation in the test. The reasonable consistency with the test data confirms the capability and accuracy of the numerical model in assessing the keying and diving of OMNI-Max anchors in clay soil.

\section{Keying, diving and soil failure mechanisms}

Figure 5 depicts the instantaneous velocity vectors during pullout of the OMNI-Max anchor for the same case of Fig. 4. It shows the soil failure mechanisms at three different drag distances related to the identified three main stages: (a) rapid loading; (b) keying; (c) diving. At the beginning of keying (Fig. 5(a); between point 2 and point 3 in Fig. 4), the soil adjacent to the anchor head fins (tip) moves significantly and faster, while the soil around to the anchor tail fins has moved marginally. This indicates that the anchor rotates or keying occurs at this stage. At the end of keying, as shown in Fig. 5(b) (point 3 in Fig. 4), similar soil movement can be seen around to the anchor tail fins and head fins, indicating translation of the anchor with minor rotation. In the middle of the diving stage (Fig. 5(c); point 4 in Fig. 4), the anchor keeps diving into deep soil with a constant angle, which means translation plays the dominant role at this stage.

RESULTS AND DISCUSSION: PARAMETRIC STUDY

An extensive parametric study was carried out varying (a) padeye offset ratio, $\eta$, and $(b)$ pullout angle at the padeye, $\theta_{\mathrm{a}}$. The results from this parametric study, as assembled in Tables 1 and 2, are discussed below. The soil undrained shear strength and soil sensitivity adopted are reported values $\left(s_{\mathrm{u}, \mathrm{ref}}=2 \cdot 4+1 \cdot 1 z \mathrm{kPa} ; S_{\mathrm{t}}=3\right)$ at the Gulf of Mexico (Zimmerman et al., 2009), where OMNI-Max anchors were installed. Parameters in terms of rate 


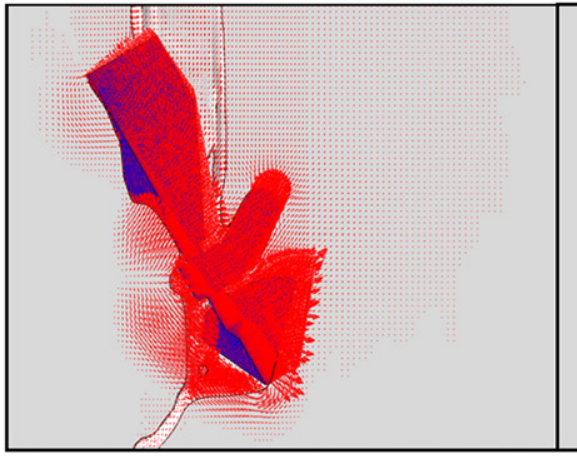

(a)

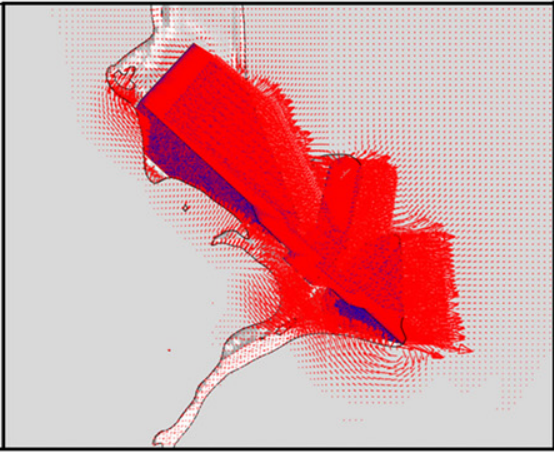

(b)

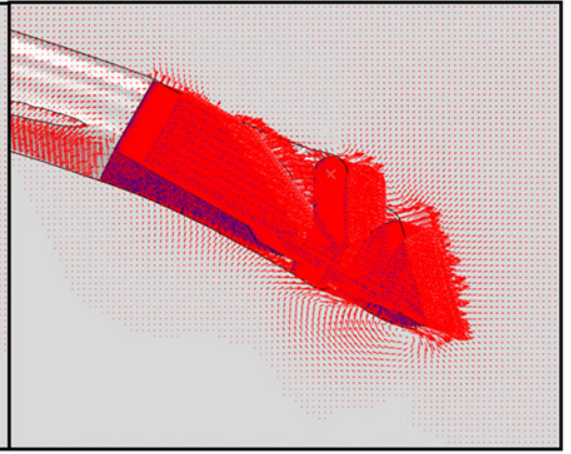

(c)

Fig. 5. Soil failure mechanism at different drag distances: (a) $u=4.6 \mathrm{~m}$; (b) $u=5.6 \mathrm{~m}$; (c) $25 \mathrm{~m}$

Table 2. Summary of 3D LDFE analyses performed

\begin{tabular}{|c|c|c|c|c|c|c|c|c|}
\hline Group & Anchor & $s_{\mathrm{u}, \mathrm{ref}}: \mathrm{kPa}$ & $v_{\mathrm{i}}: \mathrm{m} / \mathrm{s}$ & $d_{\mathrm{e}, \mathrm{t}}: \mathrm{m}$ & $\theta_{\mathrm{a}}:$ degrees & $\eta$ & $\phi:$ degrees & Note \\
\hline I & $\begin{array}{l}\text { A2 } \\
\text { A3 } \\
\text { A4 } \\
\text { A5 } \\
\text { A6 } \\
\text { A7 }\end{array}$ & $2 \cdot 4+1 \cdot 1 z$ & 19 & $\begin{array}{l}16 \cdot 9 \\
16 \cdot 86 \\
16 \cdot 81 \\
16 \cdot 78 \\
16 \cdot 83 \\
16 \cdot 83\end{array}$ & 30 & $\begin{array}{l}0 \cdot 1 \\
0 \cdot 136 \\
0 \cdot 253 \\
0 \cdot 35 \\
0 \cdot 526 \\
0 \cdot 75\end{array}$ & $\begin{array}{r}47 \cdot 2 \\
15 \cdot 8 \\
-8 \cdot 3 \\
-15 \cdot 8 \\
-16 \cdot 1 \\
-8 \cdot 2\end{array}$ & Effect of padeye offset ratio \\
\hline II & $\begin{array}{l}\text { A2 } \\
\text { A } 3 \\
\text { A4 } \\
\text { A5 } \\
\text { A6 } \\
\text { A7 }\end{array}$ & $2 \cdot 4+1 \cdot 1 z$ & 19 & $\begin{array}{l}16 \cdot 9 \\
16 \cdot 86 \\
16 \cdot 81 \\
16 \cdot 78 \\
16 \cdot 83 \\
16.83\end{array}$ & $\begin{array}{l}15 \\
45 \\
15 \\
45 \\
15 \\
45 \\
15 \\
45 \\
15 \\
45 \\
15 \\
45\end{array}$ & $\begin{array}{l}0 \cdot 1 \\
0 \cdot 136 \\
0 \cdot 253 \\
0 \cdot 35 \\
0 \cdot 526 \\
0 \cdot 75\end{array}$ & $\begin{array}{c}23 \cdot 2 \\
73 \cdot 1 \\
5 \cdot 73 \\
41 \cdot 2 \\
-22 \cdot 52 \\
25 \cdot 17 \\
-36 \cdot 2 \\
19 \cdot 8 \\
-37 \cdot 1 \\
23 \cdot 8 \\
-25 \cdot 6 \\
33 \cdot 49\end{array}$ & Effect of pullout angle \\
\hline
\end{tabular}

dependency and strain softening were taken as $\mu=0 \cdot 1$; $\dot{\gamma}_{\text {ref }}=1.5 \% / \mathrm{h} ; \xi_{95}=20$, as they provided a good match in the validation exercise.

\section{Effect of padeye offset ratio, $\eta$}

In order to investigate the effect of the padeye offset ratio on the anchor travelling path, anchors $\mathrm{A} 2 \sim \mathrm{A} 7$, with six different padeye offset ratios of $\eta=0 \cdot 1 \sim 0.75$, were installed under an identical impact velocity $\left(v_{\mathrm{i}}\right)$ of $19 \mathrm{~m} / \mathrm{s}$. The achieved embedment depths are similar $\left(d_{\mathrm{e}, \mathrm{t}}=16.78 \sim 16.9 \mathrm{~m}\right.$; group I, Table 2$)$. The anchors were then pulled out at an angle at the padeye of $\theta_{\mathrm{a}}=30^{\circ}$. Figs $6(\mathrm{a})$ and 5(b) show, respectively, the anchor trajectory at the padeye and the evolution of the anchor tip inclination angle, $\alpha_{\text {in. }}$ Fig. 6(a) confirms that the anchor keying process and potential diving are strongly dependent on the padeye offset ratio, $\eta$. For example, the anchor with a small padeye offset ratio $(\eta \leq 0.136)$ tends to slide upward. For $\eta=0.253$ to 0.75 , after keying, the anchor dives into deeper soil, with the diving tendency and penetration depth increases with increasing $\eta$. Compared to anchor A6, anchor A7 with padeye identical inclination of $\omega=20^{\circ}$ and even higher offset ratio of $\eta=0.75$ (as opposed to 0.526) dives shallowly owing to the weightless loading arm (and hence the centroid along the centre line of the anchor) considered. This range of padeye offset ratio is important for anchor design because it leads to diving, and hence gaining capacity.

Figure 6(b) shows that, as the anchor dives into the deeper soil, the anchor inclination angle reaches a plateau or a stabilised stage (see Fig. 6(b)). This means the anchor does not rotate any further, but maintains a constant trajectory. The stabilised inclination angle reduces with increasing padeye offset ratio $\eta$.

Figure 7 shows typical pullout load profiles, presented as a function of drag distance, $u$. As discussed in the section entitled 'Validation against centrifuge test data', the pullout process can be divided into three stages. However, the curve for a small padeye offset ratio $(0 \cdot 1$, no-diving case $)$ attains a plateau earlier in the absence of the diving stage, resulting in a lower pullout capacity. The pullout capacity for the dived anchor $(\eta=0.35)$ is about 1.5 times higher compared to the no-diving anchor.

\section{Effect of pullout angle}

Figure 8 shows typical anchor trajectories (for $\eta=0 \cdot 1$ and $0 \cdot 35$ ) under pullout angles, $\theta_{\mathrm{a}}=15^{\circ}, 30^{\circ}$ and $45^{\circ}$ (anchors $\mathrm{A} 2$ and A5 in groups I and II, Table 2). Following Tian et al. (2014), Fig. 8 also includes the centroid travelling direction and corresponding stabilised angle $(\phi)$ of the OMNI-Max anchor. Overall, the stabilised centroid traveling angle $\phi$ decreases with reducing the pullout angle (including negative 


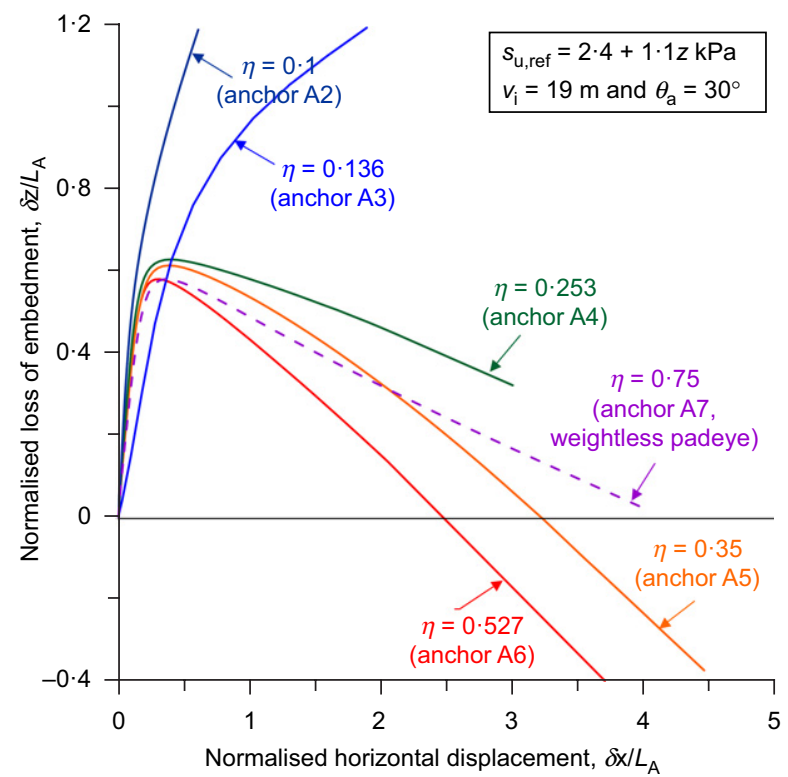

(a)

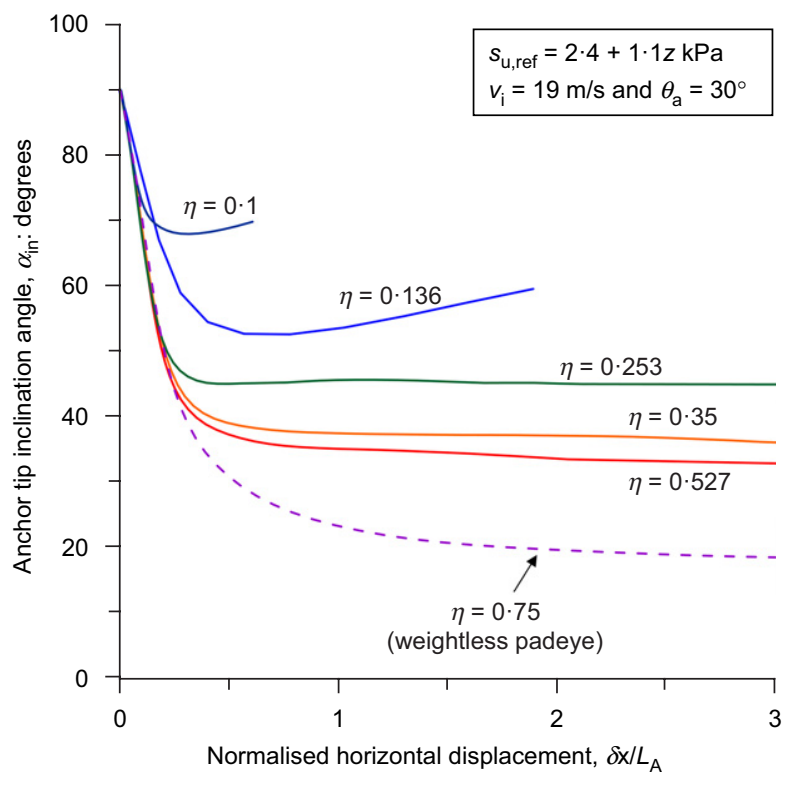

(b)

Fig. 6. Effect of padeye offset ratio (group I, Table 2): (a) anchor trajectories at padeye; (b) inclined angles at anchor tip

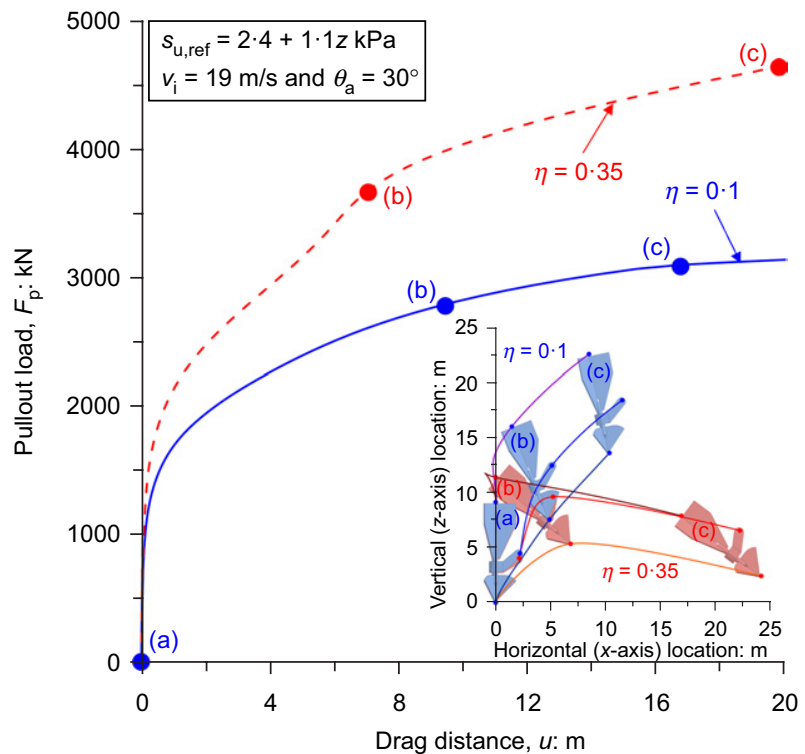

Fig. 7. Load-displacement for different padeye offset ratios (A2 and A5 in group I, Table 2)

values). For the anchor with small padeye offset ratio $(\eta=0 \cdot 1)$, smaller $\theta_{\text {a }}$ reduces the anchor lifting trend, but the anchor still travels upwards with $\phi>0$. The greater padeye offset ratio $(\eta=0 \cdot 3)$ results in an earlier transition of diving directions $(\phi<0)$ with decreasing pullout angle.

The relationship between the stabilised travelling angle, $\phi$, and the padeye offset ratio, $\eta$, is shown in Fig. 9 plotting all the results of group I $\sim$ II analyses (Table 2). The figure is divided into two areas, $\phi<0$ and $\phi>0$, each indicating when the OMNI-Max anchor will lift or dive, respectively. Three different curves displayed in the figure show a similar pattern that the anchor stabilised travelling angle $\phi$ varies with increasing padeye offset ratio $\eta$. The zone of $\phi<0$ indicates the beneficial padeye offset ratio of the OMNI-Max anchor indicating diving into deeper soil. Among all the cases, peak negative angle $\phi$ occurs for $\eta$ between 0.35 and 0.526 , which

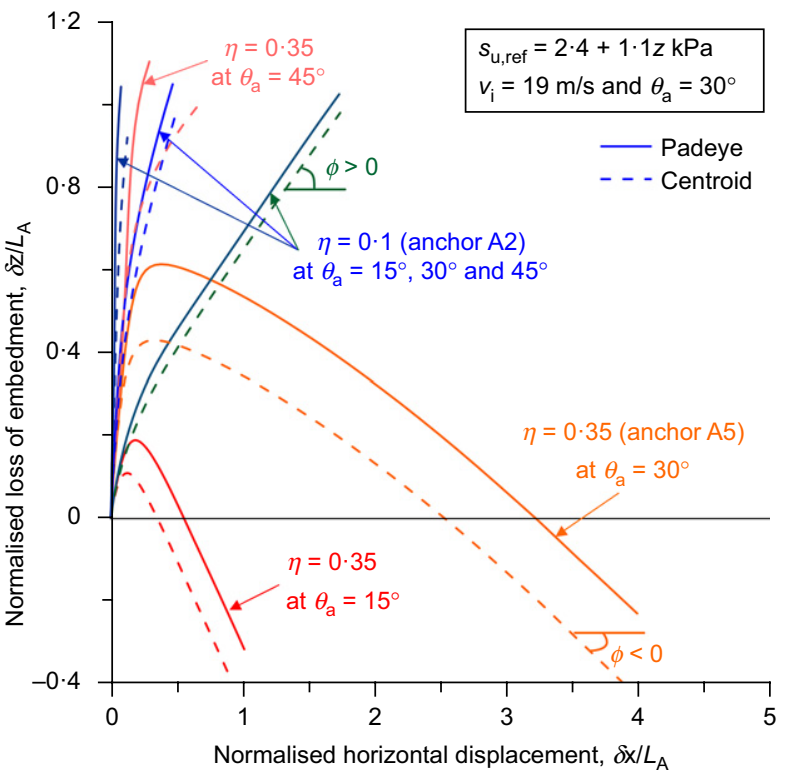

Fig. 8. Effect of pullout angle on anchor trajectories (A2 and A5 in groups I and II, Table 2)

implies that the OMNI-Max has a maximum diving trend at this padeye offset range. Furthermore, the results in Fig. 9 suggest that the anchor always lifts up for load pulling angles of more than $45^{\circ}$, regardless of the padeye offset ratio $\eta$.

\section{CONCLUDING REMARKS}

The keying process and diving potential of the dynamically installed OMNI-Max anchor were assessed through parametric 3D LDFE analyses. For the shape and weight of the considered OMNI-Max anchor (as was installed in the Gulf of Mexico) it can be recommended that the anchor diving will be ensured if the padeye offset ratio is in the range of $0 \cdot 25 \sim 0.53$ (offset angle of $14^{\circ} \sim 28^{\circ}$ ) and the chain loading 


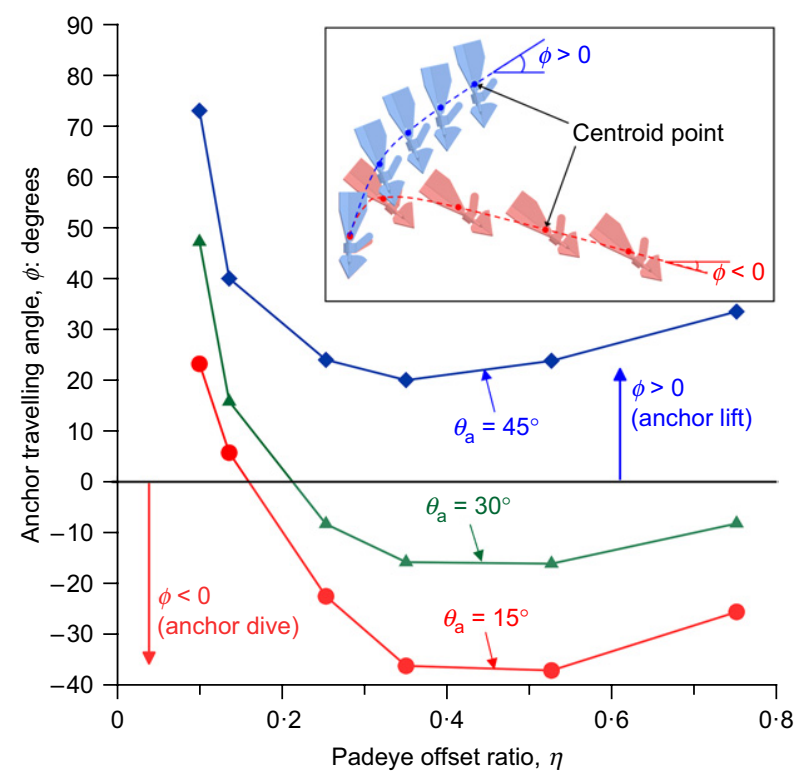

Fig. 9. Anchor travelling angle with different padeye offset ratios (groups I and II, Table 2)

inclination at the padeye $<45^{\circ}$. Anchor diving led to increasing pullout capacity. Further investigation will be carried out, varying the anchor weight and the soil strength gradient.

\section{ACKNOWLEDGEMENTS}

The research presented here was undertaken with support from the Australian Research Council (ARC) through the Discover Early Career Researcher Award (DECRA) DE140100903. The work forms part of the activities of the Centre for Offshore Foundation Systems (COFS), currently supported as a node of the Australian Research Council Centre of Excellence for Geotechnical Science and Engineering and as a Centre of Excellence by the Lloyd's Register Foundation. This support is gratefully acknowledged, as is the benefit of discussion with Dr Dong Wang.

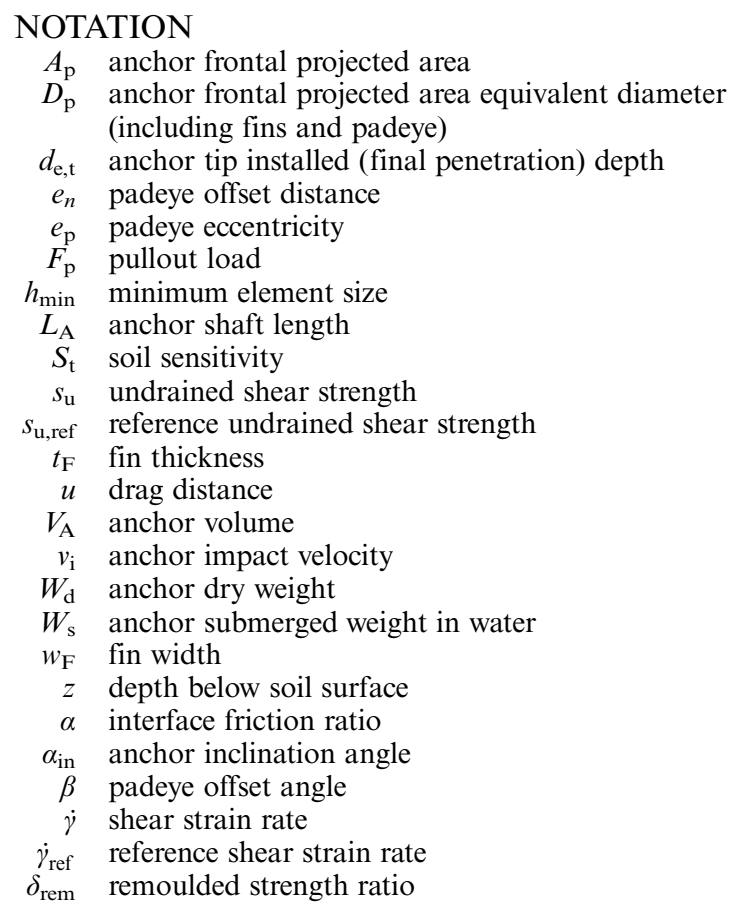

$\delta_{x}$ horizontal displacement

$\delta_{y} \quad$ vertical displacement

$\eta$ padeye offset ratio

$\theta_{\mathrm{a}}$ pullout angle at padeye

$\theta_{0} \quad$ pullout angle at mudline

$\mu$ rate parameter

$\mu_{\mathrm{c}} \quad$ Coulomb friction coefficient

$\xi$ cumulative plastic shear strain

$\xi_{95}$ cumulative plastic shear strain required for $95 \%$ remoulding

$\tau_{\max }$ limiting shear strength at soil-anchor interface

$\phi$ anchor travelling angle

$\omega$ padeye inclination angle

\section{REFERENCES}

Dassault Systèmes (2012). ABAQUS, version 6.12 EF documentation. Providence, RI, USA: Hibbitt, Karlsson \& Sorensen, Inc.

Einav, I. \& Randolph, M. (2005). Combining upper bound and strain path methods for evaluating penetration resistance. Int. J. Numer. Methods Engng 63, No. 14, 1991-2016.

Gaudin, C., O'Loughlin, C. D., Hossain, M. S. \& Zimmerman, E. H. (2013). The performance of dynamically embedded anchors in calcareous silt. Proceedings of the 32nd international conference on ocean, offshore and arctic engineering, Nantes, France, paper no. OMAE 2013-10115.

Hossain, M. S. \& Randolph, M. F. (2009). Effect of strain rate and strain softening on the penetration resistance of spudcan foundations on clay. Int. J. Geomech. 9, No. 3, 122-132.

Kim, Y. H. \& Hossain, M. S. (2015). Dynamic Installation of OMNI-Max anchors in clay: numerical analysis. Géotechnique 65, No. 12, 1029-1037, http://dx.doi.org/10.1680/jgeot.15.T.008.

Kim, Y. H., Hossain, M. S. \& Wang, D. (2014). Numerical modelling of dynamic installation of a torpedo anchor in calcareous silt. Proceedings of the international ocean and polar engineering conference, Busan, Korea (eds J. S. Chung, F. Vorpahl, S.-W. Hong, S. Y. Hong, T. Kokkinis and A. M. Wang), pp. 687-692. Cupertino, CA, USA: International Society of Offshore and Polar Engineers (ISOPE).

Kim, Y. H., Hossain, M. S., Wang, D. \& Randolph, M. F. (2015a). Numerical investigation of dynamic installation of torpedo anchors in clay. Ocean Engng 108, 820-832.

Kim, Y. H., Hossain, M. S. \& Wang, D. (2015b). Effect of strain rate and strain softening on the embedment depth of a dynamically installing anchor in clay. Ocean Engng 108, 704-715.

Liu, J., Lu, L. \& Yu, L. (2014). Large deformation finite element analysis of gravity installed anchors in clay. Proceedings of the $33 r d$ international conference on ocean, offshore and arctic engineering, San Francisco, CA, USA, paper no. OMAE 2014-24347.

Low, H. E., Randolph, M. F., DeJong, J. T. \& Yafrate, N. J. (2008). Variable rate full-flow penetration tests in intact and remoulded soil. Proceedings of the 3rd international conference on site characterization, Taipei, Taiwan, pp. 1087-1092.

Lunne, T. \& Andersen, K. H. (2007). Soft clay shear strength parameters for deepwater geotechnical design. Keynote address. Proceedings of the 6th international offshore site investigation and geotechnics conference, London, UK, pp. 151-176.

Ma, J., Wang, D. \& Randolph, M. F. (2014). A new contact algorithm in the material point method for geotechnical simulations. International Journal for Numerical and Analytical Methods in Geomechanics 38, No. 11, 1197-1210.

Neubecker, S. R. \& Randolph, M. F. (1995). Profile and frictional capacity of embedded anchor chains. J. Geotech. Engng 121, No. $11,797-803$

Nie, C. \& Shelton, J. (2011). Prediction of gravity installed anchors installation penetration. Proceedings of Oceans' 11 MTS/IEEE KONA, IEEE, Hawaii, pp. 1845-1849.

Randolph, M. F. (2004). Characterisation of soft sediments for offshore applications. Proceedings of 2nd international conference on geotechnical and geophysical site characterisation, Porto, Portugal, vol. 1, pp. 209-231.

Shelton, J. T. (2007). Omni-Max anchor development and technology. Proceedings of the oceans conference, IEEE, Vancouver, Canada, pp. 1-10.

Shelton, J. T., Nie, C. \& Shuler, D. (2011). Installation penetration of gravity installed plate anchors-laboratory study results and field 
history data. Proceedings of the offshore technology conference, Houston, TX, USA, paper OTC 22502.

Tian, Y., Cassidy, M. J. \& Gaudin, C. (2014). The influence of padeye offset on plate anchor re-embedding behaviour. Géotechnique Lett. 4, No. 1, 39-44.

Tian, Y., Gaudin, C., Randolph, M. F. \& Cassidy, M. J. (2015). The influence of padeye offset on the bearing capacity of three dimensional plate anchors. Can. Geotech. J. 52, No. 6, 682-693.

Wei, Q., Tian, Y., Cassidy, M. J., Gaudin, C. \& O’Loughlin, C. D. (2015). Behaviour of OMNI-Max anchors under chain loading. Proceedings of the $3 \mathrm{rd}$ international symposium on frontiers in offshore geotechnics, ISFOG, Oslo, Norway, pp. 925-930.

Zheng, J., Hossain, M. S. \& Wang, D. (2015). New design approach for spudcan penetration in nonuniform clay with an interbedded stiff layer. J. Geotech. Geoenviron. Engng, ASCE 141, No. 4, 04015003-1-04015003-8.

Zimmerman, E. H., Smith, M. W. \& Shelton, J. T. (2009). Efficient gravity installed anchor for deep water mooring. Proceedings of the offshore technology conference, Houston, TX, USA, paper OTC 20117. 